\title{
Intervenciones Preventivas para el Manejo de la Hipertensión Arterial en Colombia 1998-2005
}

\author{
Producing know-how and making recommendations for promoting \\ high blood pressure management in Colombia, 1998-2005
}

\author{
Jesús A. Ortega-Bolaños \\ Secretaría Distrital de Salud de Bogotá, Colombia. jaortega@saludcapital.gov.co \\ Recibido 20 Junio 2007/Enviado para Modificación 31 Diciembre 2007/Aceptado30 Marzo 2008
}

\section{RESUMEN}

Objetivo Generar conocimientos y recomendaciones sobre las intervenciones comunitarias más efectivas en Promoción de la Salud para el manejo de la Hipertensión Arterial en Colombia.

Métodos Se realizo una revisión sistemática en las bases de datos Cochrane, Lilacs, Ovid, Proquest y Pubmed, cuyo interés sobre el objeto de estudio se enmarcó en las intervenciones comunitarias más efectivas a nivel mundial para el manejo de la hipertensión arterial. La pregunta de investigación permitió incluir como palabras de búsqueda: Revisión sistemática, intervención comunitaria, costo efectividad, promoción salud, hipertensión. Se revisaron estudios en español, ingles y portugués.

Las estrategias de investigación utilizadas partieron de definir el enfoque metodológico más pertinente para involucrar el nivel individual, interpersonal y comunitario en el desarrollo del proyecto.

Resultados Se obtuvieron 1041 artículos de revisiones sistemáticas y revisión de revisiones, 246 Abstracs, 197 artículos sobre intervenciones educativas para prevenir y controlar la hipertensión arterial y 53 artículos con diferentes enfoques de intervenciones informativas y de comunicación. De este universo de información solo 11 artículos completos referenciados cumplieron con los niveles de evidencia y criterios de evaluación necesarios para generar recomendaciones

Conclusiones La evidencia sobre programas efectivos y culturalmente apropiados para fomentar la disminución de estos factores de riesgo es limitada. Se requiere mayor evidencia sobre intervenciones comunitarias dirigidas a grupos poblacionales vulnerables, adaptadas a las características culturales en los ámbitos cotidianos en los cuales viven con sus familias e involucren determinantes sociales relacionados con su estado de salud.

Palabras Clave: Literatura de revisión, costo efectividad, promoción de la salud, hipertensión (fuente: DeCS, BIREME). 


\begin{abstract}
Objective Producing know-how and making recommendations concerning the most effective health promotion community interventions for managing high blood pressure in Colombia.

Methods A systematic review was made of the Cochrane, Lilacs, Ovid, Proquest and Pubmed databases, the main interest of the study lying within the framework of the most effective community interventions around the world for managing high blood pressure. The following search words were used: systematic review, community intervention, cost effectiveness, health promotion and high blood pressure. Studies published in Spanish, English and Portuguese were reviewed. The research strategies used were derived from defining the most pertinent methodological approach for involving individual, interpersonal and community levels in developing the project.
\end{abstract}

Results 1041 articles were obtained from the systematic review of the literature: 246 abstracts, 197 articles about educational interventions for preventing and controlling high blood pressure and 53 articles adopting different approaches regarding informative interventions and communication. Only 11 complete referenced articles from this world of information fulfilled the levels of evidence and evaluation criteria necessary for producing recommendations.

Conclusions The available evidence concerning effective, culturally-suitable programmes and for promoting a reduction in these risk factors is limited. Greater evidence regarding community interventions is required for reducing risk factors directed towards special population groups adapted to the cultural characteristics of the participating population. This must involve determinants of the social and physical context related to social practices, these being developed on a large scale within the daily settings in which the subjects and their families are living.

Key Words: Systematic review, cost effectiveness, health promotion, high blood pressure (source: MeSH, NLM).

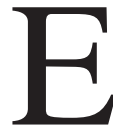

n el ámbito mundial la mayoría de las muertes se deben a enfermedades no transmisibles (32 millones), y de éstas más de la mitad (16,7 millones) son imputables a las enfermedades cardiovasculares (ECV); más de una tercera parte de esas defunciones se dan en adultos de mediana edad (1). En 1997, dichos eventos produjeron más de 10 millones de muertes y se espera que para el 2005 dicha cifra alcance los 20 millones anuales, de los cuales alrededor del 65 \% serán en países en vías de desarrollo y en la región de Europa Oriental $(2,3)$.

Los principales factores de riesgo de enfermedades cardiovasculares a nivel mundial (4) -consumo de tabaco, alimentación inadecuada y falta de actividad física (concentraciones de lípidos poco saludables, un alto índice de 
masa corporal e hipertensión arterial) (5)-son la causa del 75 \% al 85 \% de los nuevos casos de cardiopatía coronaria $(1,6)$.

Además, la mortalidad en el mundo por enfermedades cardiovasculares es el resultado de una combinación de estas causas (7). En conjunto, el colesterol origina más de cuatro millones de muertes prematuras al año, el tabaco casi cinco millones y la hipertensión arterial 7,1 millones, cifra que representa aproximadamente el $13 \%$ de la mortalidad total a nivel mundial $(8,9)$.

Es en la enfermedad Cardiocerebrovascular en la cual se tiene más oportunidad de incidir mediante un control adecuado de la Tensión arterial (10), puesto que los estudios controlados muestran reducciones de la mortalidad de hasta un $40 \%$ en adultos $(11,4)$. Cabría valorar si esta misma situación se produce en los países en vías de desarrollo, si estas desigualdades están dadas por diferencias en la incidencia de la enfermedad hipertensiva (12), por diferencias en la prevalencia de factores de riesgo primarios, o si hay inequidades en el acceso a los servicios de salud para el control y prevención de estos factores de riesgo $(13,14)$.

En Colombia, las ECV representaron la principal causa de mortalidad en mayores de 45 años, con una carga de enfermedad de 20,5 años de vida saludables perdidos (AVISAS) por cada 1000 durante el periodo 1985-1995(6,15). Durante el mismo periodo la Hipertensión arterial se considero un indicador de riesgo para la presentación de ECV, siendo el evento que produce una alta morbilidad(16), una gran letalidad y discapacidad $(17,18)$ generando un numero alto de AVISAS (12,6\% del total de años de vida saludables perdidos) y representando cerca del $30 \%$ del total de muertes $(13,19)$.

Se carece actualmente en nuestro medio del conocimiento actualizado sobre el manejo de la Hipertensión Arterial (20); por lo cual el objetivo de esta revisión sistemática de literatura científica es presentar con un buen nivel de evidencia las mejores recomendaciones y conocimientos que puedan ser acogidos por los diferentes actores del SGSSS para el manejo de la hipertensión arterial en Colombia.

\section{METODOLOGÍA}

Una Revisión sistemática (21-22) sobre las intervenciones comunitarias más efectivas a nivel preventivo para el manejo de la hipertensión arterial. Se contó con dos revisores para la construcción del protocolo y la estrategia de búsque- 
da-PICOT (1. Población general de ambos géneros sin distinciones de razaniños, niñas, adolescentes y jovenes entre 5 y 24 años, 2. Intervenciones educativas; 3.Comparación con intervenciones de información y comunicación; 4. Desenlaces o resultados, y; 5. Tipos de estudio iniciando con ensayos clínicos o comunitarios, luego cohortes, casos y controles y, posteriormente, estudios de prevalencia o series de casos).

Se realizo la búsqueda en las bases de datos de Cochrane, Lilacs, Ovid, Proquest y Pubmed incluyendo los términos MeSH (Medical Subject Heading): Revisión sistemática, intervención comunitaria, costo efectividad, promoción salud, Hipertensión (23,24). Se seleccionaron los artículos publicados originales desde Enero de 1998 hasta Junio de 2005. No hubo limitaciones de idioma ni lugar de residencia o zona geográfica $(25,26)$. Se establecieron límites solo a artículos de revistas científicas y textos completos disponibles.

Se obtuvieron 1041 referencias de artículos de revisiones sistemáticas y revisión de revisiones, identificando 246 Abstracs de los cuales 197 correspondían a artículos sobre intervenciones educativas para prevenir y controlar la hipertensión arterial y 53 artículos planteaban diferentes enfoques de intervenciones informativas y de comunicación incluyéndose únicamente 11 artículos completos $(27,28)$.

Se complementó este proceso con la revisión directa de cada una de las referencias que citaban las publicaciones encontradas en las bases de datos para identificar artículos que pudieran haberse omitido en la búsqueda inicial $(29,30)$.

Se determinaron los criterios para calificar los niveles de evidencia de los desenlaces obtenidos con la estrategia de búsqueda y de este análisis se definieron las 11 revisiones que soportan las recomendaciones que se presentan en este articulo.

\section{RESULTADOS}

Los resultados mostraron un solo artículo de metanálisis, que corresponde al 9,1\%, valorado en la categoría A; el 90,9\% de las intervenciones revisadas en 10 artículos correspondieron a tres revisión de revisiones y a siete revisiones sistemáticas que cumplieron con aspectos metodológicos necesarios para ser clasificados en la categoría B, que se presentan en la Tabla 1. 
1. En los países desarrollados las estrategias poblacionales orientadas a reducir la tensión arterial son costo-eficaces. La legislación puede ser más costoeficaz que los acuerdos voluntarios con la industria - efecto basado en suponer que la promulgación y aplicación de leyes logrará reducir más la ingesta alimentaría de sal que los acuerdos voluntarios (31)-pero es probable que la elección de una u otra opción dependa del contexto territorial (32).

Tabla 1. Resultados de evaluación y categorización de evidencia. Bogota 1998 - 2005.

\begin{tabular}{|c|c|c|}
\hline Autores & Nivel & Categoria \\
\hline X. Xin, J. He, 2001; (34) & $\begin{array}{l}\text { Nivel I. metan alisis } \\
\text { de ensa yos clínicos } \\
\text { controla dos y } \\
\text { aleatorizados }\end{array}$ & $\begin{array}{l}\text { - Buena (categoría A): existe adecuada } \\
\text { evidencia dentífica para recomendar o } \\
\text { desaconsejar la adopción de la } \\
\text { intervención comunitaria. }\end{array}$ \\
\hline $\begin{array}{l}\text { Svetkey LP, E rlinger TP ,2001;(35) } \\
\text { Shepherd J, Garcia J ,2001;(38) } \\
\text { Shephe rd J, Re becca Rees ,2001;(41) } \\
\text { Clare Blackburn, Nick Spencer, 2003; } \\
\text { (43) }\end{array}$ & $\begin{array}{l}\text { Nivel III. Ensayos } \\
\text { controla dos y } \\
\text { aleatorizados de } \\
\text { mu estra pequ eña }\end{array}$ & $\begin{array}{l}\text { - Regular (categoria B): existe cierta } \\
\text { evidencia cientifica (no conduyente) } \\
\text { para recomendar o desaconsejar la } \\
\text { adop ción de la intervención comunitaria. }\end{array}$ \\
\hline $\begin{array}{l}\text { Rebe } \propto \text { ca Rees, Angela Harden ,2001;(37) } \\
\text { Cam pbell NR, Burgess E ,2000;(42) } \\
\text { Miller ER 3rd, Erlinger TP ,2004; (33) }\end{array}$ & $\begin{array}{l}\text { Nivel IV. Ensayos } \\
\text { prospectivos } \\
\text { controlados no } \\
\text { aleatorizados }\end{array}$ & $\begin{array}{l}\text { - Regular (categoria B): existe derta } \\
\text { evidencia dentifica (no conduyente) } \\
\text { para recomendar o desaconsejar la } \\
\text { adopdón de la intervención comunitaria. }\end{array}$ \\
\hline $\begin{array}{l}\text { Appel LJ.,2003; (39) } \\
\text { Shamir R, Fisher EA, 2000; (48) }\end{array}$ & $\begin{array}{l}\text { Nivel V. Ensayos } \\
\text { prospectivos } \\
\text { controlados no } \\
\text { aleatorizados }\end{array}$ & $\begin{array}{l}\text { - Regular (categoria B): existe cierta } \\
\text { evidencia cientifica (no conduyente) } \\
\text { para recomendar o desaconsejar la } \\
\text { adop dón de la intervención comunitaria. }\end{array}$ \\
\hline Lambert A, Sargent JD, 2004; (46) & $\begin{array}{l}\text { Nivel V. Estudio de } \\
\text { cohorte }\end{array}$ & $\begin{array}{l}\text { - Regular (categoria B): existe cierta } \\
\text { evidencia dentífica (no conduyente) } \\
\text { para recomendar o desaconsejar la } \\
\text { adop coón de la intervención comunitaria. }\end{array}$ \\
\hline
\end{tabular}

2. Los resultados de las intervenciones para promover el ejercicio y la buena nutrición mostraron cambios favorables en la conducta, estado de salud física y funcionamiento de promoción de la salud escolar (33). De otra parte los resultados muestran variaciones por edad, genero, con mujeres jóvenes que empiezan a ser más receptivas a estas iniciativas (34).

3. Las mujeres jóvenes expresaron considerable insatisfacción con la organización, enseñanza y facilidades disponibles para participar en las actividades físicas en el colegio y manifiestan que su punto de vista no ha sido tenido en cuenta para mejorar la calidad de las intervenciones a implementar (35).

4. La información recogida y evaluada sobre la investigación para la promoción de alimentación saludable (32), la actividad física en grupos socialmente excluidos es reducida y por lo tanto su visión sobre las determinantes de salud e inequidades en salud $(34,36)$ no es tan fuerte para los escritores en política. 
5. Las intervenciones basadas en el colegio en las cuales educan a la gente joven acerca de la nutrición (37) y se alertan sobre la influencia ambiental y sus habilidades para la ingesta de comida saludable pueden ser efectivas pero diferenciales entre ambos sexos (32).

6. Existe amplia evidencia de la asociación de la enfermedad hipertensiva con factores de riesgo modificables; específicamente el consumo de alcohol (33), el cigarrillo $(38,39)$, la inactividad física y los inadecuados patrones de alimentación $(40,33)$.

7. Las intervenciones comunitarias sobre el consumo de frutas y verduras y la actividad física son relevantes por la creciente evidencia sobre los efectos protectores que poseen para prevenir la enfermedad hipertensiva (34).

8. Todos los programas fueron desarrollados y evaluados en colegios de secundaria, donde algunas intervenciones se iniciaron en la escuela primaria (33). Todos fueron intervenciones con multicomponentes realizados en diferentes ámbitos como el salón de clase, el colegio, el hogar usando diferentes metodologías como educación didáctica, grupos de discusión y medios masivos de comunicación (34).

9. Las intervenciones comunitarias deben ser iniciadas en poblaciones jóvenes (41).Se identificaron pocas intervenciones comunitarias realizadas en gente joven, siendo ellos los que proveen importante información que debe ser tenida en cuenta para promover la alimentación saludable, la actividad física, controlar el consumo de tabaco y alcohol como causas inmediatas para la presentación de la hipertensión arterial (40).

10. El enfoque de riesgo para la enfermedad hipertensiva y la multicausalidad inmediata están dados por los siguientes componentes:1) la carga genética, 2) las familias con factores de riesgo cardiovascular, 3) los comportamientos producto de variables intrapersonales, socioculturales y del ambiente físico (31).

11. El colegio se identificó como un área clave de realización de intervenciones para disminuir los factores de riesgo planteados para la presentación de la Hipertensión arterial (31) y requiere para su éxito el concurso de profesores y estudiantes en el diseño y operación de las intervenciones con enfoque preventivo y de acuerdo a su particularidad (41). 
12. Las intervenciones comunitarias realizadas en los colegios han comenzado a tener un mayor desarrollo por su costo efectividad (38), la cual esta limitada por la evidencia sobre la evaluación rigurosa de calidad de las propuestas comunitarias, tanto en los países desarrollados como en vías de desarrollo (36).

13. Los estudios deben realizarse con lo que la gente joven plantea, pero no se profundiza en este aspecto ni se promueve estilos de vida saludables que permitan disminuir la presentación de la Hipertensión arterial (39). Los estudios que involucran los puntos de vista de gente joven tienen que definir como los involucran en la planeación y proceso de toma de decisiones para el desarrollo y evaluación de intervenciones de promoción de la salud $(38,41,36)$.

14. La promoción de la actividad física ha empezado su inclusión en la Salud Publica requiriendo examinar la literatura sobre la forma de organizarse y enseñar en los colegios $(35,39,37)$.

\section{DISCUSIÓN}

Las intervenciones fueron frecuentemente limitadas en su efectividad (34). Todos los desenlaces evaluados fueron juzgados como parcialmente efectivos y solo para algunos desenlaces se evidencio potenciales explicaciones para la variabilidad en la efectividad (32).

Es de resaltar que las intervenciones educativas en comunidad que se consideraron efectivas han sido exitosas en países desarrollados por lo que su inclusión en países considerados en vía de desarrollo deben respetar e incluir en su implementación la particularidad de la población donde se planteen replicar $(42,43)$.

Los resultados del análisis de las intervenciones comunitarias sobre la hipertensión arterial y sus causas inmediatas obtenidas permitieron concluir que "la intervención comunitaria para prevenirla se puede generalizar en muchos casos, resulta costo-eficaz gracias a la participación de los medios de difusión, facilita la divulgación de los datos a través de las redes comunitarias y puede influir en las políticas ambientales, reguladoras e institucionales que configuran la salud" $(44,45)$.

Los aspectos fundamentales a tener en cuenta en el diseño de las intervenciones efectivas para prevenir la hipertensión arterial, dependen del ámbito: En el ámbito escolar las temáticas a tener en cuenta son la posibilidad de escogencia 
y autonomía, la estructura ambiental del colegio, y la relación entre estudiantes y profesores (46).

En el ámbito familiar es fundamental el rol de los amigos, la influencia de pares y la relación familiar. Se enfatiza en el aspecto económico, acceso a la informacion, seguridad y restricción de la libertad, limitaciones de tiempo y conveniencia (47). En el auto-cuidado se enfatiza el control y empoderamiento, las preferencias personales y la apariencia personal.

En América latina, la evidencia con respecto a programas efectivos y culturalmente apropiados para fomentar la disminución de estos factores de riesgo es limitada (48-50). Específicamente en Colombia se requiere de mayor evidencia con respecto a intervenciones comunitarias para disminuir los factores de riesgo que: 1)estén dirigidas a grupos poblacionales especiales (41), 2) estén adaptadas a las características culturales de la población participante (31), 3) se desarrollen a gran escala, 4) involucren determinantes del contexto social y físico relacionados con las practicas sociales de interés y , 5) se desarrollen en los ámbitos cotidianos en los cuales viven los sujetos y sus familias (34) •

\section{REFERENCIAS}

1. OMS, Informe sobre la Salud en el mundo 2002.Ginebra: OMS; 2002. Cáp. 4. p. 61.

2. Puska P. The North Karelia Project: evaluation of a comprehensive community programme for control of cardiovascular diseases in North Karelia, Finland, 1972-1977.Copenhagen, WHO Regional Office for Europe; 1981.

3. Puska P (Ed). The North Karelia Project. 20 year results and experiences. Helsinki, the National Public Health Institute, Helsinki University Printing House; 1995.

4. Puska $P(E d)$. Comprehensive cardiovascular community control programmes in Europe. Copenhagen, WHO Regional Office for Europe; 1988 (EURO Reports and Studies 106).

5. Secretaría Distrital de Salud. Dirección de Salud Pública. Series Históricas de Salud de Santa Fe de Bogotá. 1986-1996. 1a edición; Agosto 1988. pp. 47,76.

6. Ministerio de Salud. Colombia. II Estudio Nacional de Factores de Riesgo de Enfermedades Crónicas- ENFREC II. Tomo III: Perfil lipídico y conocimientos, actitudes y prácticas en hipertensión, ejercicio, hiperlipidemias y peso corporal; 1999.

7. Chobanian AV, Bakris GL. The National High Blood Pressure Education Program Coordinating Committee. The Seventh Report of the JOINT NATIONAL COMMITTEE on Prevention, Detection, Evaluation and Treatment of High Blood Pressure. JAMA 2003; 289: 2560-72.

8. Berríos X. Time trends in risk factors of chronic diseases: is a new epidemic comming? Revista Medica de Chile; 1997, 125: 1405-1407.

9. Ministerio de Protección Social-Instituto Nacional de Salud. Situación de Salud en Colombia. Indicadores Básicos 2002.Disponible en: http://www.minproteccionsocial.gov.co Consultado: 7 de septiembre de 2005.

10. Hemmelgarn BR, McAllister FA. The 2005 Canadian Hypertension Education Program recommendations for the management of hypertension: Part 1 - Blood pressure measurement, diagnosis and assessment of risk. Can J Cardiol. 2005; 21:645-56. 
11. Berrios X. Distribution and prevalence of major risk factors of non communicable diseases in selected countries: the WHO Inter-Health Programme. Bulletin of the World Health Organización. 1997; 75:99-108.

12. Williams B, Poulter NR. British Hypertension Society. British Hypertension Society guidelines for hypertension management 2004 (BHS-IV): summary. BMJ 2004; 328: 634-40.

13. Máscioli.SR, Grimm.RH. Characteristics of participants at base line. In the treatment of mild hypertension Study (TOMMHS) Am. J. Cardiol., 1990; 66: 32 C-35C.

14. Secretaría Distrital de Salud D.C.-Dirección de Salud Pública, Universidad Nacional de Colombia-Facultad de Medicina. Lineamientos de Política Pública de Prevención de las Enfermedades Crónicas en Bogotá D. C.1a edición; Mayo 2002. p. 76.

15. Indicadores de Salud en Colombia [Internet]. Disponible en: http://www.col.ops- oms.org/ sivigila/2001/bole01_2001.pdf Consultado: 7 de septiembre de 2005.

16. MacMahon B, Yen S. Coffee and cancer of the pancreas. N Engl J Med 1981; 304:630-633.

17. McMahon S, Peto R. Blood Pressure, Stroke and coronary heart disease: part I, prolonged differences blood pressure: prospective observacional studies corrected for the regression dilution bias. Lancet, 1990; 335: 765.

18. Collins R, Peto R. Blood pressure, Stroke and coronary heart disease: Part II: Short-term reductions in blood pressure: overview of randomized, drug trials in their epidemiological context. Lancet, 1990; 335: 827.

19. European Society of Hypertension-European Society of Cardiology guidelines for the management of arterial hypertension. Guidelines Committee. Journal of Hypertension 2003; 21: 1011 - 53.

20. Khan NA, McAllister FA. The 2005 Canadian Hypertension Education Program recommendations for the management of hypertension: Part II - Therapy. Can J Cardiol. 2005; 21:657-72.

21. Hedin A, Källestål C. Knowledge-based public health work Part 2. Handbook for compilation of reviews on interventions in the field of public health.National institute of public healthSweden; 2004. Cap I. p. 10.

22. Clarke M, Ox man AD. (Ed). Cochrane Reviewers' Handbook 4.2.0. [Internet]. Disponible en: http://www.cochrane.org/resources/handbook/index.htm Consultado: 19 de marzo 2005.

23. Rothman KJ, Greenland S. Modern epidemiology. 2a. edition. East Washington Square, PA: Lippincott-Raven Publishers; 1998.

24. Undertaking Systematic Reviews of Research on Effectiveness. CRD's Guidance for those Carrying Out or Commissioning Reviews. CRD Report Number 4 (2nd Edition). NHS Centre for Reviews and Dissemination, University of York. March 2001.

25. Luepker RV. Community education for cardiovascular disease prevention: risk factor changes in the Minnesota Heart Health Program. American Journal of Public Health, 1994,

84: 1383-1393.

26. Cochrane Health Promotion and Public Health Field. Victorian Health Promotion Foundation Cochrane Collaboration Open Learning Materials for Reviewers. Versión 1.1, November 2002.

http://www.cochrane.org [consultado el 19 de marzo 2005]

27. Hedin A, Kallestal C. Knowledge based public health work [Internet]. Part 2: Handbook for compilation of reviews on interventions in the field of public health. National Institute of Public Health. 2004. Disponible en: http://www.fhi.se/shop/material_pdf/ r200410Knowledgebased2.pdf Consultado: 21 de marzo 2005.

28. Bero LA, Grilli R, Grimshaw JMI. Closing the gap between research and practice: an overview of systematic reviews of interventions to promote the implementation of research findings. BMJ 1998; 317:465-468.

29. Bandura A. Social Learning Theory. New Jersey: Prentice Hall; 1977.

30. Vartiainen $\mathrm{E}$. Changes in risk factors explain changes in mortality from ischemic heart disease in Finland.British Medical Journal, 1994, 309: 23-27. 
31. Miller ER 3rd, Erlinger TP .Results of the Diet, Exercise, and Weight Loss Intervention trial (DEWIT).Welch Center for Prevention, Epidemiology and Clinical Research, USA: The Johns Hopkins Medical Institutions,Baltimore, MD;2004.

32. Shepherd J, Rebecca R. Young people and Healthy eating: a systematic review of research on barriers and facilitators. Report EPPI-Centre; October 2001.

33. Svetkey LP, Erlinger TP. Effect of lifestyle modifications on blood pressure by race, sex, hypertension status, and age. Duke Hypertension Center and the Sarah W Stedman Nutrition and Metabolism Center. USA: Duke University Medical Center, Durham, NC; 2001.

34. Shepherd J, Garcia J. Barriers to, and Facilitators of, the Health of Young People: A systematic review of evidence on young people's views and on interventions in mental health, physical activity and healthy eating. Report EPPI-Centre; October 2001.

35. Rees R, Harden A. Young people and physical activity: a systematic review of research on barriers and facilitators. Report EPPI-Centre; October 2001.

36. Appel LJ. Professor of Medicine. Lifestyle modification as a means to prevent and treat high blood pressure. Welch Center for Prevention, Epidemiology and Clinical Research, Johns Hopkins Medical Institutions, Baltimore, Maryland, USA. J Am Soc Nephrol. 2003; 14: S99-S102.

37. Shamir R, Fisher EA, Dietary therapy for children with hypercholesterolemia. Bruce Rappaport School of Medicine, Haifa, Israel. Am Fam Physician 2000; 61:675-82,685-6.

38. Blackburn C, Spencer N. Effect of strategies to reduce exposure of infants to environmental tobacco smoke in the home: cross sectional survey BMJ-2003; 327; 257.

39. Lambert A, Sargent JD. How Philip Morris unlocked the Japanese cigarette market: lessons for global tobacco control. San Francisco, USA. Tobacco Control 2004; 13:379-387.

40. X. Xin, J. He. Effects of Alcohol Reduction on Blood Pressure: A Meta-Analysis of Randomized Controlled Trials. Hypertension, November 1, 2001; 38(5): 1112 - 1117.

41. Campbell NR, Burgess E. Methods and overview of the Canadian recommendations. Canadian Hypertension Society, Canadian Coalition for High Blood Pressure Prevention and Control, Laboratory Centre for Disease Control at Health Canada, Heart and Stroke Foundation of Canada; 2000

42. Kanell WB, Gordon TE. Evaluation of cardiovascular risk in the elderly: the Framingham Study. Bull NY Acad Med 1978; 54: 573-591.

43. Karvonen M. Prehistory of the North Karelia Project. In: Puska P, Tuomilehto J, Nissinen A, Vartiainen E (Ed). The North Karelia Project: 20 year results and experiences. Helsinki: The National Public Health Institute. 1995: 17-21.

44. Brunner E. Can dietary interventions change diet and cardiovascular risk factors? A metaanalysis of randomized controlled trials. American Journal of Public Health, 1997; 87: 415-422.

45. Kannel WB. Some lessons in cardiovascular epidemiology from Framingham. American Journal of Cardiology. 1976; 37: 269.

46. Moore LL, Nguyen US. Preschool physical activity level and change in body fatness in young children. The Framingham Children's Study. Am J Epidemiol, 1995; 142:982-988.

47. Tian H-G. Changes in sodium intake and blood pressure in a community-based intervention project in China. Journal of Human Hypertension. 1995; 9:959-968.

48. Schooler C. Synthesis of findings and issues from community prevention trials. Annals of Epidemiology, 1997, 57: 554-568.

49. Jackson N. Systematic Reviews of Health Promotion and Public Health interventions. A Public Health Education and Research Project funded by the Australian Government Department of Health and Ageing; 2002.Unit Two. p. 7.

50. Kelsey JL, Thompson WD. Methods in observational epidemiology. Nueva York: Oxford University Press, 1986. 